\title{
A Novel Five-coordinate Mn Complex with a Redox-active SNNNS Ligand System
}

\author{
Katharina Dürr ${ }^{\mathrm{a}}$, Denis A. Yalalov ${ }^{\mathrm{b}}$, Frank W. Heinemann ${ }^{\mathrm{c}}$, Svetlana B. Tsogoeva ${ }^{\mathrm{b}}$, \\ and Ivana Ivanović-Burmazovića \\ ${ }^{a}$ Lehrstuhl für Bioanorganische Chemie, Department Chemie und Pharmazie, Universität Erlangen- \\ Nürnberg, Egerlandstraße 1, 91058 Erlangen, Germany \\ b Organische Chemie, Department Chemie und Pharmazie, Universität Erlangen-Nürnberg, \\ Henkestraße 42, 91054 Erlangen, Germany \\ ${ }^{c}$ Anorganische und Allgemeine Chemie, Department Chemie und Pharmazie, Universität Erlangen- \\ Nürnberg, Egerlandstraße 1, 91058 Erlangen, Germany
}

Reprint requests to Prof. Dr. I. Ivanović-Burmazović. Fax: (+49) 91318527387.

E-mail: ivana.ivanovic@chemie.uni-erlangen.de

Z. Naturforsch. 2010, 65b, 258-262; received December 23, 2009

Dedicated to Professor Rolf N. Saalfrank on occasion of his $70^{\text {th }}$ birthday

\begin{abstract}
Synthesis and characterization of a manganese complex of a new redox-active diaminocyclohexane derivative of a thiourea-based ligand with SNNNS donor atom set is reported. The molecular structure of the complex exhibits a pentadentate SNNNS ligand coordinated in the neutral form to the $\mathrm{Mn}(\mathrm{II})$ center. The coordination around the central metal is best described as distorted trigonal bipyramidal with the pyridine nitrogen and the two sulfur atoms being in the equatorial plane and the remaining two nitrogen donors occupying the apical positions of the bipyramid. Cyclic voltammetry in DMSO shows the $\mathrm{Mn}^{\mathrm{II}} / \mathrm{Mn}^{\mathrm{III}}$ redox couple with the oxidation peak at $-0.199 \mathrm{~V}$ and the reduction peak at $-0.587 \mathrm{~V} v s$. $\mathrm{Fc} / \mathrm{Fc}^{+}$(scan rate $0.5 \mathrm{~V} \mathrm{~s}^{-1}$ ). The ligand itself possesses redox activity and exhibits prominent oxidation and reduction peaks at -1.194 and $-1.481 \mathrm{~V} v s$. $\mathrm{Fc} / \mathrm{Fc}^{+}$, respectively. The results of electrochemical experiments under oxygen atmosphere suggest that upon reaction with electrochemically generated superoxide the redox-active ligand is modified causing decomposition of the complex.
\end{abstract}

Key words: Manganese, Five-coordinate Complexes, SNNNS Ligation, Superoxide

\section{Introduction}

In our research on model complexes of superoxide dismutase (SOD) [1,2], we have been working mostly with seven-coordinate macrocyclic manganese and iron complexes with the ligand systems containing nitrogen and oxygen donor atoms [3-5]. It has been suggested in the literature that high SOD activity is provided by N5-macrocyclic ligands with high conformational flexibility (Fig. 1) [6-8]. This conclusion results from a correlation between the ability of the seven-coordinate complex to adopt a six-coordinate (pseudo)octahedral geometry (upon water release) and SOD efficiency, predominantly based on MM calculations [7]. If this mechanism applies, acyclic complexes with even higher flexibility should result in greater SOD activity. Therefore, we became interested in designing an acyclic ligand system analogous to the one of the proven macrocyclic SOD mimetic

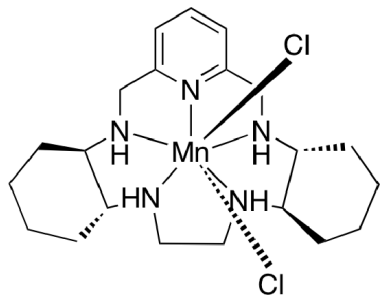

M 40403
Fig. 1. Structure of SOD mimetic M 40403.
M40403 (Fig. 1), which has already entered clinical trials. To achieve that goal, we chose to work with new diaminocyclohexane derivatives [9] as ligand fragments, which in reaction with a pyridine derivative yield an acyclic pentadentate ligand framework (Eq. 1). The acyclic character of such ligands will enable easier conformational rearrangements around metal centers. Additionally, these diaminocyclohexane derivatives are used in enantioselective organocataly- 


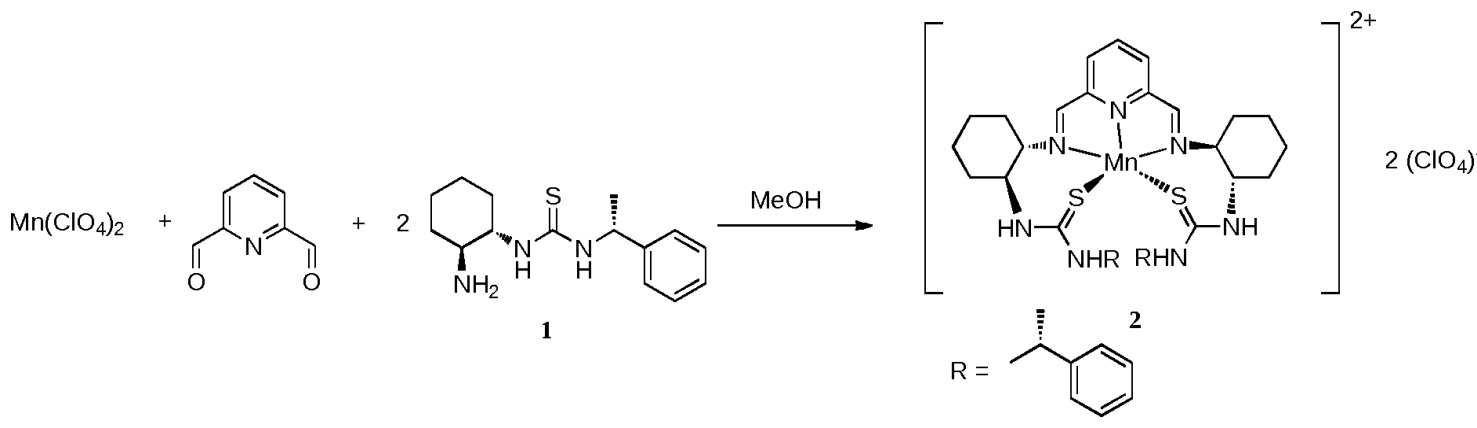

sis [9]. In order to explore the coordination behavior of this ligand class, we started working with the thioureabased ligand fragment (Eq. 1). Complexes with coordinated sulfur are not expected to be SOD-active. However, such studies can provide valuable structural information which can be used for further development of analogous complexes where sulfur is replaced by oxygen. Complexes with the SNNNS bonding pattern are not very common in the literature. The only related pyridine-derived systems known in the literature are complexes of 2,6-diacetylpyridinebis $\left(\mathrm{N}^{4}\right.$-azacyclic thiosemicarbazones) $[10,11]$. They have been prepared with several types of metal ions, among them Mn(II). They also show thione-thiol tautomerism, which is not possible with our ligand system.

\section{Results and Discussion}

In this study, we synthesized and characterized a novel manganese complex with an SNNNS coordination motif. Similar to the preparation of other sevencoordinate complexes $[4,5]$ we reacted the aminethiourea ligand fragment [9] and pyridine-2,6-dicarbaldehyde together with manganese(II) perchlorate in $\mathrm{MeOH}$ solution in a template synthesis (Eq. 1). X-Ray quality crystals of $\mathbf{2}$ could be obtained.

The molecular structure of $\mathbf{2}$ (Fig. 2) exhibits a pentadentate SNNNS ligand coordinated in the neutral form to the $\mathrm{Mn}$ (II) center. The coordination is best described as distorted trigonal bipyramidal with the pyridine nitrogen and the two sulfur atoms in the equatorial plane and the remaining two nitrogen donors representing the apical positions of the bipyramid. The manganese and the three nitrogen donor atoms are almost exactly coplanar. The complex cation carries two positive charges, and electroneutrality is accomplished by two perchlorate groups as non-coordinating anions. For the pyridyl ring in the middle of the two

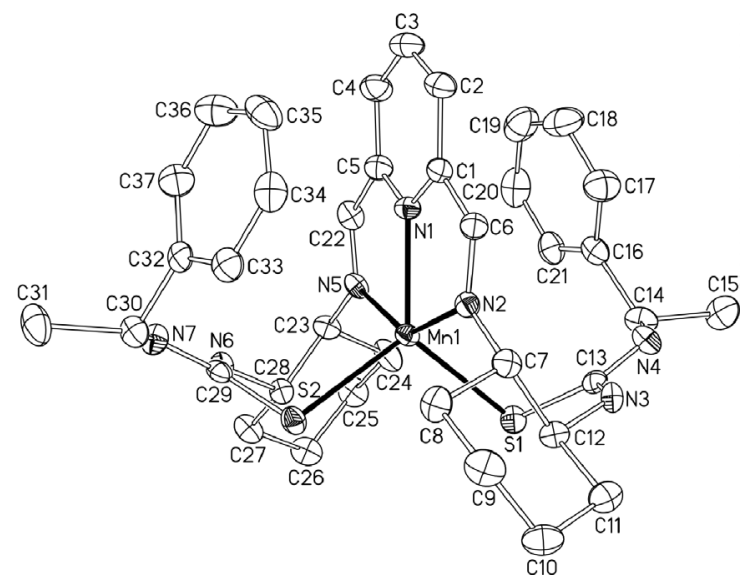

Fig. 2. Displacement ellipsoid representation of the cation of $250 \%$ probability ellipsoids, hydrogen atoms omitted for clarity). Selected bond lengths (A, e. s. d.'s in parentheses): Mn1-S1 2.4619(9), Mn1-S2 2.4285(9), Mn1-N1 2.140(3), Mn1-N2 2.313(2), Mn1-N5 2.305(3) A. Selected bond angles (deg., e.s.d.'s in parentheses): N1-Mn1-S1 128.22(7), N1-Mn1-S2 124.16(7), S1-Mn1-S2 107.60(3), N2-Mn1N5 145.72(9).

phenyl rings, a parallel arrangement is realized indicating $\pi-\pi$ stacking of the three rings with a distance between them of around $4 \AA$. This is at the longer end of typical $\pi-\pi$ interactions, but typical for pyridine arene $\pi-\pi$ interactions [12]. The stacking is enabled by the electron-poor nature of the metal-coordinated pyridine ring [12]. The centers of the phenyl rings centers are positioned exactly on top of the pyridyl nitrogen atom. This most probably is a stabilizing factor for the whole molecular structure. The complex cation exhibits approximately two-fold symmetry with the noncrystallographic twofold axis going through the metal atom and the nitrogen atom of the pyridine ring.

Cyclic voltammetry of $\mathbf{2}$ was performed in DMSO solution in order to determine the redox properties of the complex. The cyclic voltammogram of $\mathbf{2}$ in nitrogen-purged DMSO solution is shown in Fig. 3. 


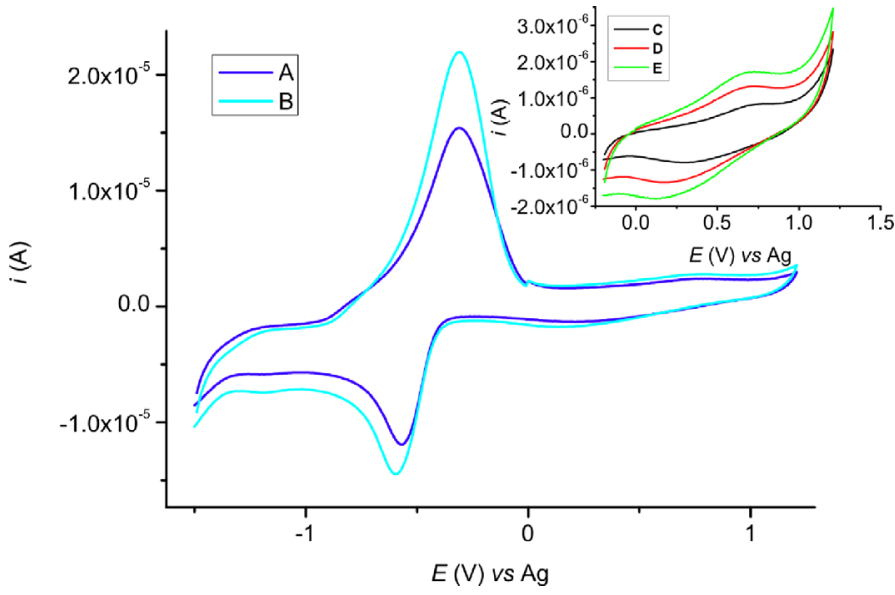

Fig. 3. Cyclic voltammogram of $\mathbf{2}$ in DMSO purged with nitrogen at $25^{\circ} \mathrm{C}$. Conditions: conc. of 2: $10^{-3} \mathrm{~mol} \mathrm{~L}^{-1}$, conc. of $\mathrm{Bu}_{4} \mathrm{NPF}_{6}: 0.1 \mathrm{~mol}$ $\mathrm{L}^{-1}$, scan rates: $\mathrm{A}: 1.0 \mathrm{~V} \mathrm{~s}^{-1}$ and $\mathrm{B}: 1.5 \mathrm{~V} \mathrm{~s}^{-1}$; inset: cyclic voltammogram of positive region. Conditions: conc. of 2: $10^{-3} \mathrm{~mol} \mathrm{~L}^{-1}$, conc. of $\mathrm{Bu}_{4} \mathrm{NPF}_{6}$ : $0.1 \mathrm{~mol} \mathrm{~L}^{-1}$, scan rates: C: $0.1 \mathrm{~V} \mathrm{~s}^{-1}$, D: $1.0 \mathrm{~V} \mathrm{~s}^{-1}$ and $\mathrm{E}: 1.5 \mathrm{~V} \mathrm{~s}^{-1}$.

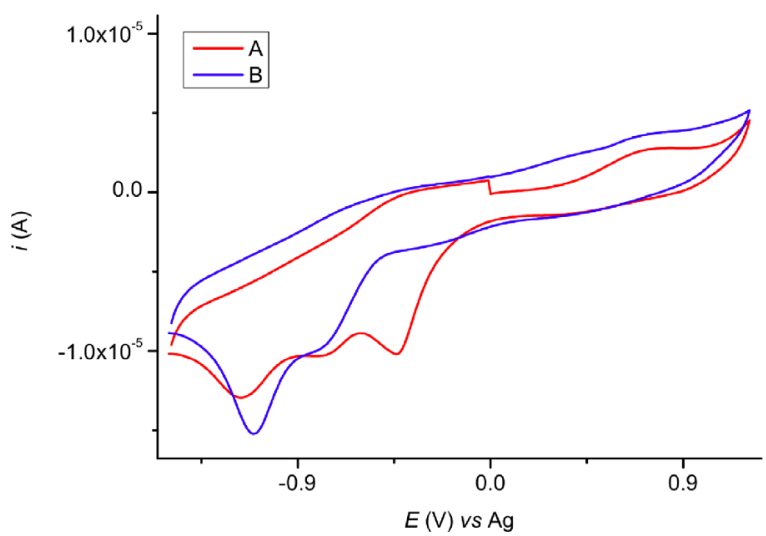

Fig. 4. Cyclic voltammogram of $\mathbf{2}$ in DMSO at $25^{\circ} \mathrm{C}$. Conditions: conc. of $2: 10^{-3} \mathrm{~mol} \mathrm{~L}^{-1}$, conc. of $\mathrm{Bu}_{4} \mathrm{NPF}_{6}: 0.1 \mathrm{~mol}$ $\mathrm{L}^{-1}$, scan rate: $0.5 \mathrm{~V} \mathrm{~s}^{-1}$; A: purged with oxygen, 1 scan; B: purged with oxygen, 2 scans.

The sulfur-containing ligand possesses redox activity and exhibits prominent oxidation and reduction peaks at -1.194 and $-1.481 \mathrm{~V}$ vs. $\mathrm{Fc} / \mathrm{Fc}^{+}$(scan rate $0.5 \mathrm{~V} \mathrm{~s}^{-1}$ ), respectively. Complex 2 also shows metalcentered redox processes. The $\mathrm{Mn}^{\mathrm{II}} / \mathrm{Mn}^{\mathrm{III}}$ redox couple appears in the positive region of the cyclic voltammogram (inset in Fig. 3) with the oxidation peak at $-0.199 \mathrm{~V}$ and the reduction peak at $-0.587 \mathrm{~V} v s$. $\mathrm{Fc} / \mathrm{Fc}^{+}$(scan rate $0.5 \mathrm{~V} \mathrm{~s}^{-1}$ ). Such values and the low intensity of the corresponding peaks are typical for manganese(II) complexes.

The solution of $\mathbf{2}$ was also purged with oxygen in order to determine the reactivity of the complex towards electrochemically generated superoxide, as oxygen is reduced with formation of superoxide at negative potentials. Going to negative potential, the reduc- tion of oxygen to superoxide and ligand reduction are observed. The ligand reduction occurs at more negative potentials than it is the case under nitrogen atmosphere, and it proceeds in two steps (Fig. 4). The scan in the oxidation direction does not show reoxidation peaks for oxygen and the ligand, suggesting that upon a reaction with superoxide the ligand was chemically modified. A second scan under the same conditions shows disappearance of the metal redox couple. Such behavior is probably due to the decomposition of the complex and binding of the ligand or its fragments to the surface of the gold working electrode, since in general sulfur compounds tend to bind to gold surfaces.

\section{Conclusion}

We have synthesized and characterized a fivecoordinate Mn(II) complex containing the SNNNS donor atom set. Due to the steric hindrance induced by the diaminocyclohexane-thiourea ligand, coordination of an additional sixth ligand is not favored. The stronger donor ability of the sulfur atom competes successfully with the donor ability of the secondary amino groups and enables formation of sevenmembered chelate rings, different from the common five-membered chelate rings in the structure of M 40403 (Fig. 1). The results of electrochemical experiments suggest that upon reaction with superoxide the redox-active ligand is modified causing decomposition of the complex. This may be the reason why thio-ketone-containing ligand systems are in general not suitable for designing SOD mimetics. It should be mentioned that in the nickel-containing SOD enzyme $[13,14]$ and in the corresponding model complexes 
[15] two cysteinate sulfur donor atoms are involved in the coordination. A detailed study on the products of the superoxide reaction is in progress.

\section{Experimental Section}

\section{General information}

Reagents and solvents were obtained from commercial sources and were of reagent quality unless otherwise stated. All chemicals were used as received without further purification. The synthesis of ligand fragment $\mathbf{1}$ has been published elsewhere [9]. IR spectra were recorded on an ATI Mattson FTIR $60 \mathrm{AR}$ spectrometer. Elemental analysis was done on a Carlo Erba EA $1108 \mathrm{CHN}$ instrument. UV/Vis spectra were taken on a Hewlett-Packard 8452A spectrophotometer.

\section{Cyclic voltammetry}

Cyclic voltammetric measurements were carried out using an Autolab instrument with a PGSTAT 30 potentiostat. A conventional three-electrode arrangement was employed consisting of a gold working disk electrode (Metrohm, geometric area: $0.07 \mathrm{~cm}^{2}$ ), a platinum wire (Metrohm) as the auxiliary electrode, and silver wire as pseudo-reference electrode. All measurements were done in DMSO in the presence of $0.1 \mathrm{M}$ tetrabutylammonium hexafluorophosphate as supporting electrolyte. The $\mathrm{Fc}^{+} / \mathrm{Fc}(\mathrm{Fc}=$ ferrocene) couple was used to calibrate the redox potentials. All solutions without superoxide were thoroughly degassed with nitrogen prior to the beginning of the experiments, and during the measurements a nitrogen atmosphere was maintained. Measurements with superoxide were carried out by saturating the solution with dry air.

Safety Notes! Perchlorate salts of metal complexes with organic ligands are potentially explosive. Only a small amount of material should be prepared and handled with great care.

\section{Synthesis of 2}

A solution of $100 \mathrm{mg} 1(0.361 \mathrm{mmol})$ and $24.3 \mathrm{mg}$ pyridine-2,6-dicarbaldehyde $(0.180 \mathrm{mmol})$ in methanol $(20 \mathrm{~mL})$ was stirred at $55{ }^{\circ} \mathrm{C}$ for $1 \mathrm{~h}$. Then a solution of $65.2 \mathrm{mg} \mathrm{Mn}\left(\mathrm{ClO}_{4}\right)_{2} \cdot 6 \mathrm{H}_{2} \mathrm{O}(0.181 \mathrm{mmol})$ in methanol ( $2 \mathrm{~mL}$ ) was added to give a color change to orange. After $1 \mathrm{~h}$ of stirring, the solvent was removed. The crude mixture was taken up in acetonitrile and filtered. Orange crystals suitable for X-ray crystallography were obtained by crystallization from methanol/acetonitrile/diethyl ether/ hexane.

Yield: $115 \mathrm{mg}(70 \%)$ orange needles. - UV/Vis (DMSO): $\lambda\left(\varepsilon\right.$ in $\left.\mathrm{L} \mathrm{mol}^{-1} \mathrm{~cm}^{-1}\right)=297$ (15200), $312 \mathrm{~nm}$ (10200). - IR (KBr): $v=3316,3080$, 2935,
Table 1. Crystal data, data collection and structure refinement details for $\mathbf{2}$.

\begin{tabular}{ll}
\hline Formula & $\mathrm{C}_{38} \mathrm{H}_{49.50} \mathrm{Cl}_{2} \mathrm{MnN}_{7.50} \mathrm{O}_{8.50} \mathrm{~S}_{2}$ \\
$M_{\mathrm{r}}$ & 937.31 \\
Crystal size, $\mathrm{mm}^{3}$ & $0.35 \times 0.28 \times 0.11$ \\
Crystal system & monoclinic \\
Space group & $P 2_{1}$ \\
Temperature, $\mathrm{K}$ & $150(2)$ \\
$a, \AA$ & $11.547(1)$ \\
$b, \AA$ & $23.138(2)$ \\
$c, \AA$ & $17.183(2)$ \\
$\beta$, deg & $107.661(8)$ \\
$V, \AA^{3}$ & $4374.5(7)$ \\
$Z$ & 4 \\
$D_{\text {calcd }}, \mathrm{mg} \mathrm{m}^{-3}$ & 1.42 \\
$\mu\left(\right.$ Mo $\left.K_{\alpha}\right), \mathrm{mm}^{-1}$ & 0.6 \\
$F(000), \mathrm{e}$ & 1956 \\
$h k l$ range & $\pm 14, \pm 29, \pm 22$ \\
Refl. measured $/$ unique $/ R_{\text {int }}$ & $97603 / 19179 / 0.0579$ \\
Refl. observed $[I \geq 2 \sigma(I)]$ & 15697 \\
Param. refined & 1105 \\
$R_{1}(F)[I \geq 2 \sigma(I)]$ & 0.0432 \\
$w R_{2}\left(F^{2}\right)$ (all refls.) & 0.0930 \\
Absolute structure parameter $[16]$ & $0.005(11)$ \\
$\Delta \rho_{\text {fin }}($ max $/$ min $)$, e $\AA^{-3}$ & $0.84 /-0.52$ \\
\hline
\end{tabular}

2861, 1569, 1495, 1450, 1376, 1349, 1286, 1241, 1212, 1099, 705, $624 \mathrm{~cm}^{-1}$. - MS (ESI): $\mathrm{m} / \mathrm{z}(\%)=680.9$ (100) $[\mathrm{M}+\text { ligand }]^{2+}, 807.2(71)\left[\mathrm{M}+\mathrm{ClO}_{4}{ }^{-}\right]^{2+}, 354.8(43)$ $[\mathrm{M}]^{2+} .-\mathrm{C}_{37.5} \mathrm{H}_{51} \mathrm{Cl}_{2} \mathrm{MnN}_{7} \mathrm{O}_{9.5} \mathrm{~S}_{2}$ (941.82): calcd. C 47.82, H 5.46, N 10.41, S 6.47; found C 48.1, H 5.2, N 10.5, S 6.5.

\section{Crystal structure determination}

Details of the crystal structure determination are given in Table 1 . Intensity data for $\mathbf{2}$ were collected on a BrukerNonius KappaCCD diffractometer using $\mathrm{Mo} K_{\alpha}$ radiation ( $\lambda=0.71073 \AA$, graphite monochromator). Data were corrected for Lorentz and polarization effects. Absorption has been taken into account by a semi-empirical correction based on multiple scans using SADABS [17]. The structure was solved by Direct Methods and was refined on $F^{2}$ using SHELXTL NT (v6.10) [18]. All non-hydrogen atoms were refined anisotropically. Hydrogen atoms were placed in calculated positions using a riding model. The asymmetric unit of $\mathbf{2}$ contains two symmetry-independent molecules of the complex salt and one $\mathrm{MeCN}$ as well as one $\mathrm{H}_{2} \mathrm{O}$ molecule. No hydrogen atoms were included for the solvate water molecule. One of the perchlorate anions is disordered. Two preferred orientations could be refined resulting in site occupancies of 61.9(5) \% for O41-O44 and 38.1(5) \% for $\mathrm{O} 41^{\prime}-\mathrm{O} 44^{\prime}$.

CCDC 756071 contains the supplementary crystallographic data for this paper. These data can be obtained free of charge from The Cambridge Crystallographic Data Centre via www.ccdc.cam.ac.uk/data_request/cif. 
Acknowledgement

The authors gratefully acknowledge financial support from the Deutsche Forschungsgemeinschaft through SFB 583 "Redox-active metal complexes" (K.D., S.B.T. and I. I.-B.).

[1] J.P. Emerson, E.D. Coulter, D.E. Cabelli, R.S. Phillips, D. M. Kurtz, Jr., Biochemistry 2002, 41, 4348 4357.

[2] I. Fridovich, Ann. N. Y. Acad. Sci. 1999, 893, 13-18.

[3] A. Dees, A. Zahl, R. Puchta, N. J. R. Van Eikema Hommes, F. W. Heinemann, I. Ivanovic-Burmazovic, Inorg. Chem. 2007, 46, 2459-2470.

[4] G.-F. Liu, K. Duerr, R. Puchta, F. W. Heinemann, R. van Eldik, I. Ivanovíc-Burmazovíc, Dalton Trans. 2009, $6292-6295$

[5] G.-F. Liu, M. Filipovic, F. W. Heinemann, I. IvanovícBurmazovíc, Inorg. Chem. 2007, 46, 8825-8835.

[6] D. P. Riley, Chem. Rev. 1999, 99, 2573 - 2587.

[7] D. P. Riley, O.F. Schall, Adv. Inorg. Chem. 2007, 59, $233-263$.

[8] D. Salvemini, Z.-Q. Wang, J. L. Zweier, A. Samouilov, H. MacArthur, T. P. Misko, M. G. Currie, S. Cuzzocrea, J. A. Sikorski, D. P. Riley, Science 1999, 286, 304-306.

[9] D. A. Yalalov, S. B. Tsogoeva, T. E. Shubina, I. M. Martynova, T. Clark, Angew. Chem. 2008, 120, 6726-6730; Angew. Chem. Int. Ed. 2008, 47, 6624-6628.
[10] M. Mohan, P. Sharma, M. Kumar, N. K. Jha, Inorg. Chim. Acta 1986, 125, 9-15.

[11] M. Mohan, A. Agarawal, N. K. Jha, J. Inorg. Biochem. 1988, 34, $41-54$.

[12] C. Janiak, Dalton Trans. 2000, 3885 - 3896.

[13] J. Wuerges, J.-W. Lee, Y.-I. Yim, H.-S. Yim, S.-O. Kang, K.D. Carugo, Proc. Natl. Acad. Sci. U.S.A. 2004, 101, 8569-8574.

[14] D. P. Barondeau, C. J. Kassmann, C. K. Bruns, J. A. Tainer, E. D. Getzoff, Biochemistry 2004, 43, 8038 8047.

[15] M.E. Krause, A. M. Glass, T. A. Jackson, J. S. Laurence, Inorg. Chem. 2010, 49, $362-364$.

[16] H. D. Flack, Acta Crystallogr. 1983, A39, 876-881.

[17] G. M. Sheldrick, SADABS, Program for Empirical Absorption Correction of Area Detector Data, University of Göttingen, Göttingen (Germany) 2002.

[18] G. M. Sheldrick, SHELXTL NT (version 6.10), Bruker Analytical X-ray Instruments Inc., Madison, Wiscon$\sin$ (USA) 2002. See also: G. M. Sheldrick, Acta Crystallogr. 2008, A64, 112 - 122. 